\title{
Human evolution: the non-coding revolution
}

\author{
Lucía F. Franchini ${ }^{1}$ and Katherine S. Pollard ${ }^{2,3^{*}}$
}

\begin{abstract}
What made us human? Gene expression changes clearly played a significant part in human evolution, but pinpointing the causal regulatory mutations is hard. Comparative genomics enabled the identification of human accelerated regions (HARs) and other humanspecific genome sequences. The major challenge in the past decade has been to link diverged sequences to uniquely human biology. This review discusses approaches to this problem, progress made at the molecular level, and prospects for moving towards genetic causes for uniquely human biology.
\end{abstract}

\section{Post-genomic challenges for determining uniquely human biology}

When the human genome was first sequenced [1,2], the big question was "how many genes do we have?" Most people guessed too high. Sequencing our closest living relative, the chimpanzee $[3,4]$, begged the question "which genes are different?" Here the answer was predicted a century before and supported by King and Wilson's 1975 discovery that certain blood proteins have very few amino acid differences between human and chimpanzee $[5,6]$. We now know that the vast majority of all genomic changes that happened since the humanchimpanzee ancestor are in non-coding regions, consistent with King and Wilson's hypothesis that regulatory changes drove the differences between our species. In hindsight, the importance of gene regulation in human evolution is logical. There are many more DNA bases in regulatory regions than in protein-coding genes, making them a larger target for evolutionary innovation. Furthermore, genes frequently function in many different

\footnotetext{
* Correspondence: kpollard@gladstone.ucsf.edu

${ }^{2}$ Gladstone Institutes, San Francisco, CA 94158, USA

${ }^{3}$ Department of Epidemiology \& Biostatistics, Institute for Human Genetics,

Institute for Computational Health Sciences, University of California, San

Francisco, CA 94158, USA

Full list of author information is available at the end of the article
}

contexts, and this pleiotropy constrains their evolution compared to regulatory elements, which tend to be more modular [7]. Thus, regulatory sequences have great potential to be drivers of human evolution.

The challenge in the post-genomic era has been to determine which of the millions of human-specific noncoding sequence differences are responsible for the unique aspects of our biology. This is a hard problem for many reasons. First, the non-coding genome is vast, requiring methods to prioritize the mutations that matter. The neutral theory of molecular evolution, coupled with redundancy in biological networks, suggests that many human-specific DNA changes had little effect on our biology. Second, we know much less about how sequence determines function of regulatory elements compared to protein or RNA genes. Hence it is difficult to predict the molecular, cellular, and organismal consequences of human-specific regulatory mutations. Furthermore, most uniquely human traits are complex, and there is no doubt that they are encoded by a combination of mutations in different genomic loci. Finally, because gene regulation has diverged significantly between primates and model organisms such as mice, zebrafish or flies, it is hard to test hypotheses about the functional effects of regulatory mutations. In this review, we discuss advances to address these barriers with an emphasis on linking sequence to function, complementing other recent papers that explore genetic and regulatory changes in human evolution [8-13].

\section{Discovering the fastest evolving regions in the human genome}

Single nucleotide changes can have functional consequences, but currently these are difficult to predict in non-coding regions where small mutations are frequently tolerated and the function of a particular nucleotide is rarely known. Hence, human evolutionary genetics has mostly focused on genome regions with many humanspecific differences (reviewed in [14-17]). Human accelerated regions (HARs) are short, evolutionarily conserved 
DNA sequences that have acquired significantly more DNA substitutions than expected in the human lineage since divergence from chimpanzees. A number of studies applied different tests to identify HARs either genomewide $[18,19]$ or with protein-coding sequences specifically removed from the analysis [20-23]. We and the other authors of these studies had a common aim: to identify regulatory elements with human-specific activity (Fig. 1). These analyses started with regions conserved across nonhuman mammals in order to enrich for functional elements [24-27] and to increase power to detect acceleration (Box 1). Then they used various methods to identify a subset of conserved elements that accumulated humanspecific changes. Differences in analysis choices and available data over time (for example, species in alignments, methods used to identify conserved elements, tests for acceleration, bioinformatics filters to remove artifacts) resulted in only modest overlap between the HARs identified in different studies despite their common aim (Fig. 1). These studies also differed in whether they specifically tested for positive selection compared to a neutral model or simply identified acceleration, which could be due to a variety of evolutionary processes including mutation rate increases or loss of constraint (Box 1).
Collectively nearly 3000 non-coding HARs have been identified to date [14], representing a pool of candidates that can be searched for regulatory regions with humanspecific activity.

Insertions, deletions, duplications, and rearrangementscollectively known as structural variants (SVs) - contribute many more nucleotides to the genetic difference between humans and chimpanzees than do HARs and other single nucleotide variants. Indeed, the first differences detected between the human and chimp genomes were large SVs discovered prior to genome sequencing (for example, chromosome 2 fusion, inversions on chromosomes 1 and 18 [28]). Primates have accumulated SVs at an accelerated rate compared to other mammals, and the human genome contains numerous lineage-specific SVs $[29,30]$. These SVs contribute significantly to the emergence of new genes and gene families (reviewed in [31]). Most HAR analyses have specifically filtered out SVs due to the difficulty of accurately assembling and aligning these regions. But HARs are in fact common in recent segmental duplications [20], and we found them to be enriched near duplicated genes [32]. In addition, SVs can change gene expression and phenotypes by associating non-coding elements with genes they did not previously regulate ('enhancer hijacking', see below)

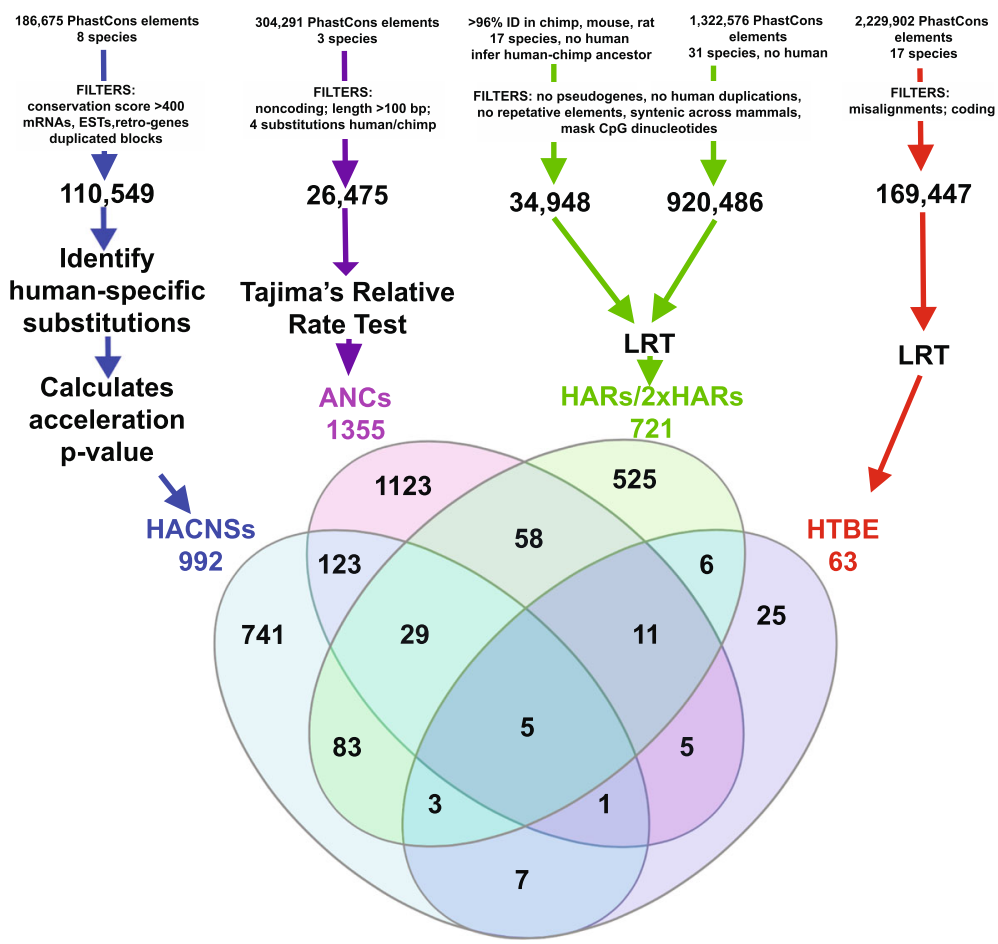

Fig. 1. Identification of human accelerated elements. Top: the four different approaches used to identify human accelerated regions. Some key differences include (i) the conserved elements used as candidates to identify HARs (which depend on multiple sequence alignments, methods to detect conservation, and whether human was masked in the alignments), (ii) bioinformatics filters that aim to restrict to non-coding elements and/or remove assembly or alignment artifacts, and (iii) tests used to detect acceleration. Bottom: overlap of the different datasets of human accelerated regions. Abbreviations: ANC accelerated conserved non-coding sequences [20]; HACNS human accelerated conserved non-coding sequences [23]; HTBE human terminal branch elements [21]. HARs include the original HARs [19] and the second generation HARs or 2xHARs [100] 


\section{Box 1 Evolutionary forces underlying the appearance of HARs}

In order to detect acceleration in a particular lineage it is necessary

to statistically test for a difference between the substitution rate

observed on that lineage and the expected rate given the rest of

the tree. The tests to detect acceleration typically use continuous

time Markov models of DNA (or protein) evolution to quantify the

likelihood of the multiple sequence alignment for a genome region

[87]. Comparing human to chimpanzee and other primates,

acceleration tests can reach genome-wide significance even when

there are only a small number of human-specific substitutions in a

100-bp region. This is especially true in regions that are conserved

across non-human primates, because the number of expected sub-

stitutions is close to zero [14]

Is acceleration equal to positive selection? Not necessarily. In order to differentiate positive selection from relaxation of constraint (both mechanisms can produce acceleration) it is important to compare the evolutionary rate of the accelerated element to the local neutral rate. Using this approach, it has been shown that many HARs ( 50\%) and haDHSs ( 90\%) are evolving faster than the neutral rate for that part of the genome, which is suggestive of adaptive evolution [88].

These divergence-based tests will detect fixed differences that largely accumulated millions of years ago. To test for recent or ongoing positive selection, one uses population genetics tests that detect reduced polymorphism relative to divergence or long haplotypes. While many sequence changes in HARs predate diversification of modern humans, some HARs do show evidence for recent selection [19, 21, 88], as do some haDHSs [38]. Another mechanism that likely shaped $\sim 20 \%$ of HARs is GC-biased gene conversion [88-90], a neutral recombination-associated process that leads to accelerated fixation of weak to strong (AT to GC) mutations. Another 20\% of HARs are not evolving faster than neutral expectations, but only faster than expected given strong conservation in other species, perhaps due to loss of functional constraint. Thus, there is not a unique underlying evolutionary mechanism that led to the appearance of all HARs. The interpretation of functional differences between orthologous sequences in HARs should consider different evolutionary forces.

$[33,34]$. Thus, further exploration of the regulatory consequences of human-specific SVs is clearly needed.

\section{Beyond conservation: combining acceleration tests and epigenetic marks}

Sequence conservation is a useful tool for predicting which non-coding substitutions will be functional. But many regulatory elements are not conserved [35, 36], and conservation does not indicate when and where a regulatory element is active. Since the initial discovery of HARs and human-specific SVs, understanding of the proteins and epigenetic marks found at particular categories of regulatory element has improved significantly, as have genome-wide functional genomics assays such as chromatin immunoprecipitation sequencing (ChIP-seq), ATAC-seq, and RNA-seq. Using these tools it is now possible to generate genome-wide maps of predicted regulatory regions across many cell types and developmental stages. We predicted enhancers in the human genome and tested 29 predictions overlapping HARs with in vivo reporter assays (see below), discovering that many of the previously identified HARs are enhancers active in the developing embryo [37].

Functional genomics data are also very useful for loosening or omitting the requirement that HARs be conserved in other species. Two recent investigations performed evolutionary analyses of putative human regulatory elements and identified many examples of human sequence acceleration missed by studies that relied on deep sequence conservation (for example, greater than $95 \%$ sequence identity across mammals or vertebrate phastCons elements). Gittelman et al. performed comprehensive evolutionary and population genetics analyses on open chromatin (specifically, DNase I hypersensitive sites (DHSs)) from 130 cell types to discover regulatory DNA with evidence of adaptive evolution in humans [38]. They discovered 524 DHSs with sequences that are conserved in non-human primates but show accelerated nucleotide substitution rates in the human lineage (haDHS). By functionally characterizing selected haDHSs using transgenic reporters and luciferase assays in different cell types, the authors identified several where the human sequence changes result in a gain of enhancer function (Table 1; Fig. 2).

Using a similar approach, Dong et al. compared substitution rates of DHSs to nearby ancestral repeats (LINE1 or LINE2 elements, assumed to be neutrally evolving) and identified 3538 accelerated DHSs (ace-DHS) [39]. Notably, only 17 accelerated DHSs overlap between these two studies, likely due to Gittelman et al. requiring conservation in primates so that they tested only 113,577 DHSs versus 808,943 in Dong et al.'s analysis, which had no such conservation filter. In addition, these two approaches used different data to estimate neutral rates for their acceleration tests. Regardless, it is clear that starting with DHSs rather than conserved elements reveals novel human-specific regulatory elements: the majority of haDHSs (454/524) and ace-DHSs (3520/ 3538) were not previously identified as HARs. It is important, however, to validate the regulatory functions of DHSs and other biochemically active genomic regions experimentally, especially if they are not conserved [36]. 


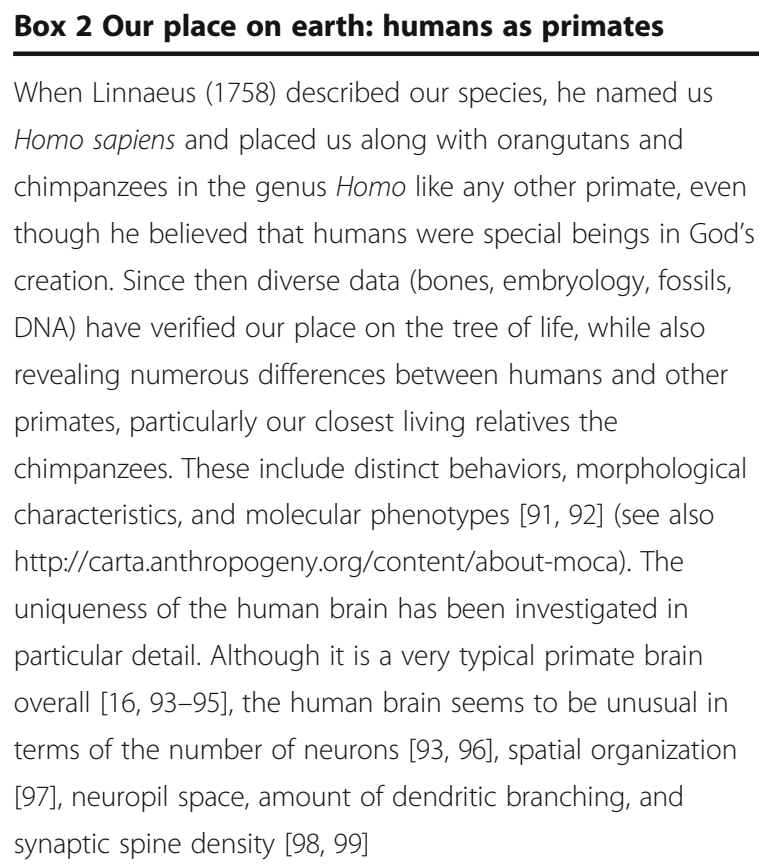

\section{Comparative identification of regulatory regions}

An alternative approach to identify human-specific regulatory elements is to perform functional genomics assays such as open chromatin or protein binding in multiple species and find regions with differential evidence of regulatory activity. Some of the first evidence that humanspecific regulatory elements may be common came from the ENCODE project, which found that a high proportion $(\sim 50 \%)$ of the biochemically active regions identified in the human genome were not conserved across mammals $[35,40]$. Several recent studies built on this observation and compared human epigenomic profiles (such as open chromatin, histone modifications) to those of primates and other species using either primary tissues or cell lines from various developmental stages (see below). To infer that regulatory marks are human-specific requires epigenomic profiles from chimpanzees (or bonobos), which is often impossible due to limited availability of material and ethical considerations. One potential solution is to investigate post hoc if the regions with epigenetic signatures that differ between human and rodents or monkeys intersect with HARs or harbor sequence changes that are unique to the human genome.

Cotney and colleagues performed ChIP-seq for histone H3 lysine 27 acetylation (H3K27ac, a mark of active enhancers) in human, rhesus macaque, and mouse embryonic limb at four different developmental stages from bud to digit separation, defined as orthologous based on morphology and HOXD gene expression [41]. They found 2175 promoters and 2915 putative enhancers with significantly higher H3K27ac signal in human at one or more time points. Sixteen of these regions overlap with HARs, but most show no evidence of human accelerated substitution rates or unusual diversity patterns across modern humans [41].

Using a similar approach, Reilly et al. mapped active promoters and enhancers using di-methylation of histone $\mathrm{H} 2$ lysine 27 (H3K27me2, a promoter mark) and H3K27ac, respectively, during three stages of human, rhesus macaque, and mouse cortex development [42]. Comparing signal for these epigenetic marks across species, the authors predicted 2855 promoters and 8996 enhancers unique to human samples (that is, with higher signal in human than other species). The majority of these regions show no evidence of human accelerated substitution rates, although 48 of them overlap HARs [42].

Vermunt and colleagues assayed H3K27ac in eight anatomical subdivisions of the adult brain (cerebellum, caudate nucleus, thalamic nuclei, putamen, white matter, precentral gyrus, prefrontal cortex, occipital pole) of human, chimpanzee and rhesus macaque [43]. The authors found that $14-43 \%$ of putative enhancers and $3-10 \%$ of promoters had differential signal between human and macaque brain regions. However, only a very small fraction of these showed a similar difference between human and chimpanzee. While 284 H3K27ac regions from this study overlap with HARs, only 32 of these have significantly higher or lower H3K27ac signal in human versus chimpanzee or macaque. One particularly interesting example is 2 xHAR87/HACNS548, which interacts with the promoter of the CADM1 gene and shows steadily increasing H3K27ac signal from macaque to human (Fig. 2).

Prescott et al. compared transcription factor (TF) and coactivator binding, histone modifications, and chromatin accessibility genome-wide in human and chimpanzee cranial neural crest cells (CNCCs) to pinpoint putative enhancers [44]. They derived CNCCs from pluripotent stem cells using an in vitro protocol in which specification, migration, and maturation are recapitulated in the dish $[45,46]$, an approach that allows unavailable chimpanzee cell types to be derived in the lab. The authors predicted $\sim 1800$ regulatory elements with different levels of H3K27ac in human versus chimpanzee, suggestive of differential enhancer activity. A few of these overlap HARs (three of the top 1000; Table 1). The authors found that the variance in H3K27ac signal scales proportionally with human-chimp sequence divergence. We note that sequence differences are quite low overall (three to six substitutions per $500 \mathrm{bp}$ ) even at regions with the biggest human-chimpanzee differences in H3K27ac binding [44]. These results show that regions with one or two sequence changes can have different enhancer activity (measured by transgenic assays using luciferase or lacZ), which may make identifying causal sequence changes easier than in HARs with five to ten changes. Prescott et al. speculate that the enhancer activity differences they detect 


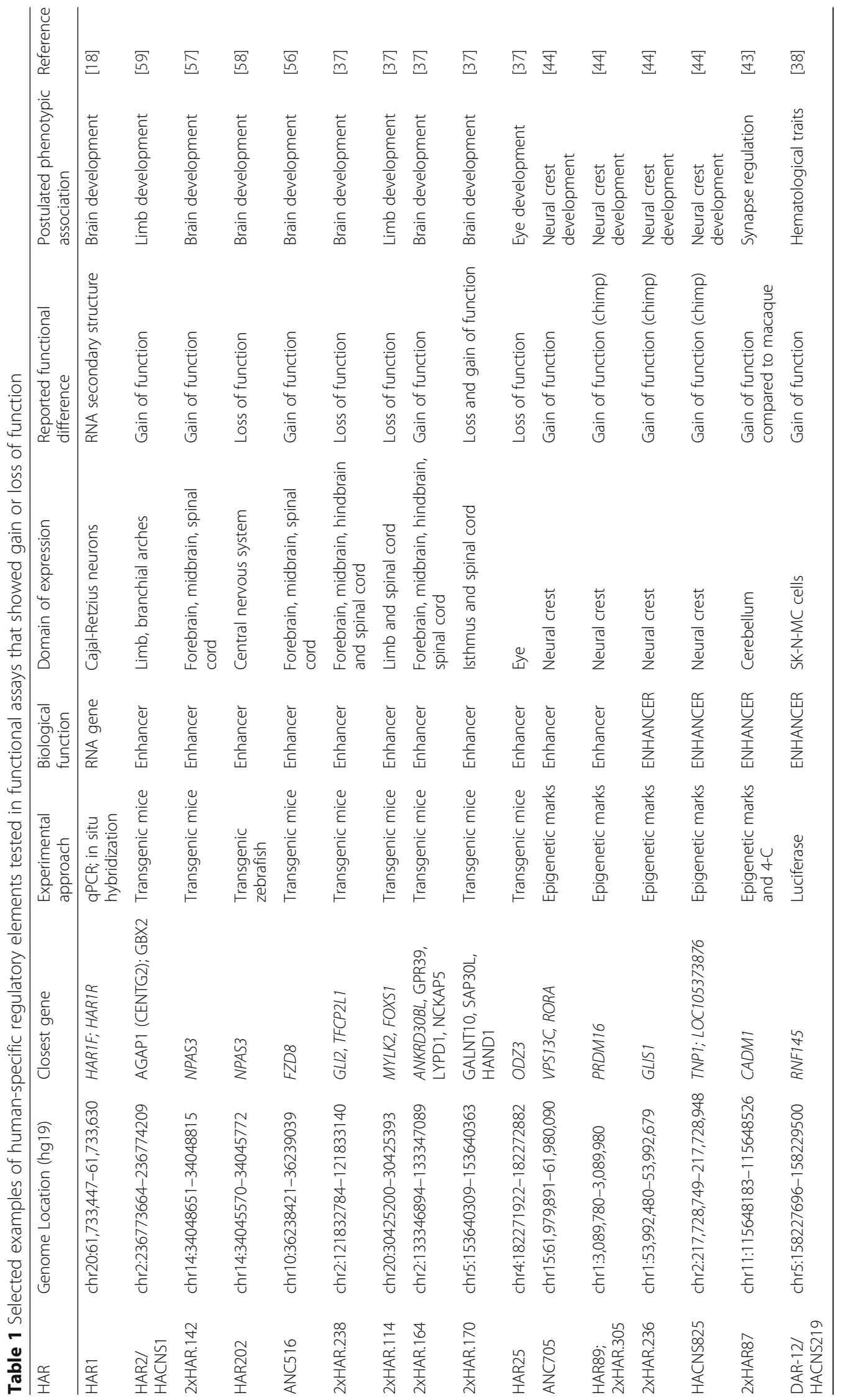




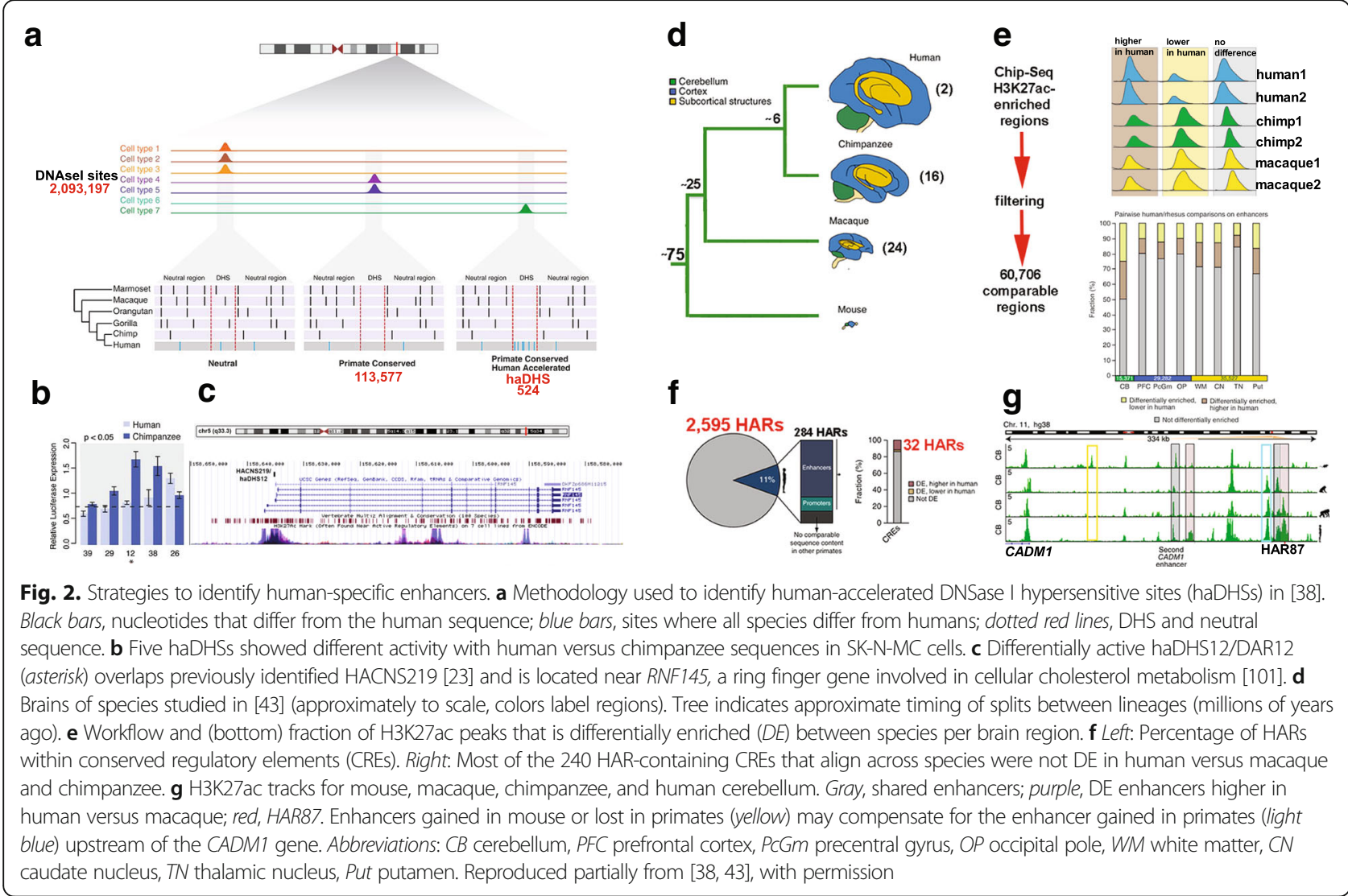

are due to changes in consensus transcription factor binding motifs, such as one called Coordinator. However, since enhancer assays are not highly quantitative (see below) and cannot identify upstream causes of differences in reporter gene activity, further functional experiments are important to validate the expression differences and test the hypothesis that transcription factor binding changes played a causal role in these regions.

Overall, these studies discovered many genome sequences with human-specific epigenomic signatures, which are exciting catalogs of putative regulatory elements for future studies. Since many of these regions are not deeply conserved across mammals, they complement previously identified HARs. However, most of the elements show little or no evidence of human-specific sequence change, suggesting that the causal mutations are in trans or that the catalogs contain many false positives due to the many challenges of cross-species functional genomics, such as antibody affinity, low samples sizes, and matching cell composition and developmental stage. As Prescott et al. note, regions with fewer sequence changes than a typical HAR can show enhancer activity differences [44], though we emphasize that it is challenging to discriminate these from the many cases where an equivalent number of sequence changes causes no activity difference. More functional validation of predicted differences in regulatory activity (for example, with reporter assays or CRISPR/Cas9 screens) will help to determine the false positive rate. Meanwhile, we should be careful when interpreting chromatin immunoprecipitation data since protein-DNA binding events cannot be directly translated into regulatory function, as has been discussed elsewhere [47-49]. Another challenge is evaluating the net effect of all genetic and epigenetic changes within a locus on species-specific gene expression (reviewed in [49]), which is particularly difficult when regulatory elements function synergistically or redundantly (Fig. 3 and below).

\section{Transgenic approaches to study the functional impact of non-coding evolution}

One approach to investigating the functional effects of human-specific non-coding sequences is to introduce them into model organisms. Despite differences in anatomy and genetic networks across species [50], transgenic and mutant mouse models have been useful for characterizing human-specific genes, such as ARHGAP11B [51] and SRGAP2C [52], and human-specific gene variants, such as FOXP2 [53, 54]. Predicted enhancers can also be validated and tested for effects of sequence differences using transient or stable reporter assays in mouse and zebrafish. In general, in transient transgenic enhancer assays (where the F0 generation is analyzed) enhancer function 


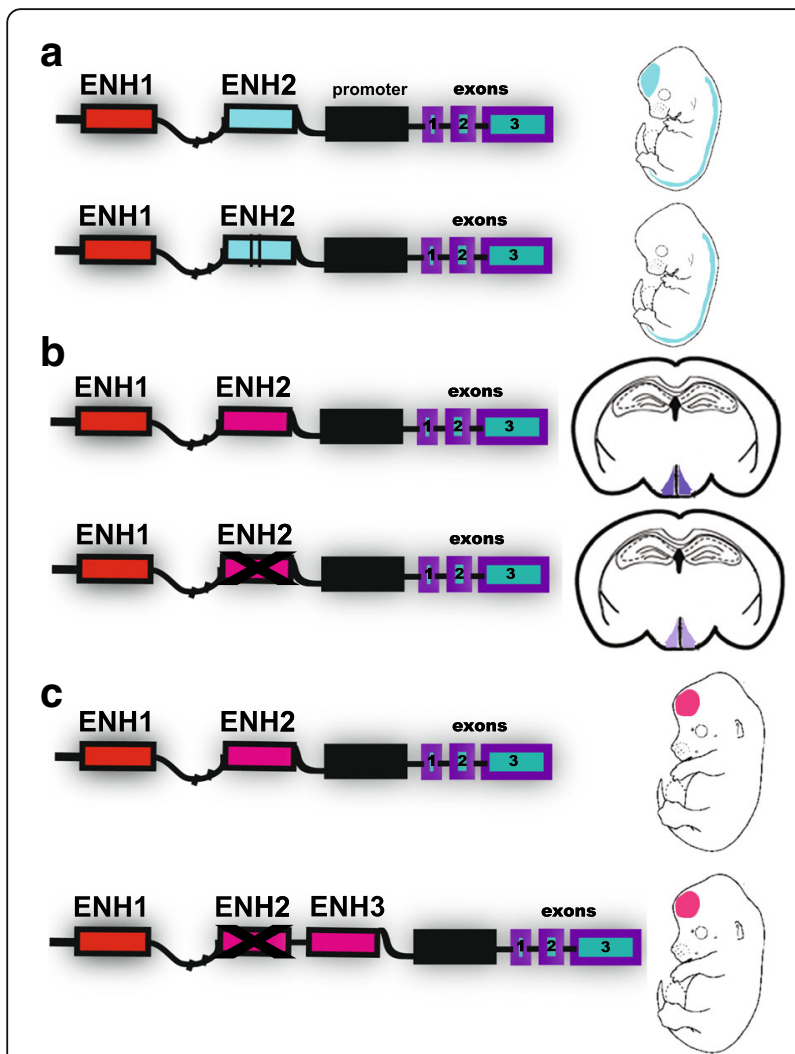

Fig. 3. Evolutionary mechanisms at the level of gene regulatory regions a An example of accelerated sequence evolution affecting one enhancer in a locus and leading to gain of an expression domain in the

developing forebrain. We characterized one such gain of function HAR in Kamm et al. [57]. b Shadow enhancers are multiple enhancers that direct a similar gene expression pattern and thus overlap in function. As such, they can act cooperatively to confer robustness in different physiological situations $[77,78]$. The example shown is based on the work of Lam et al. [78], where two enhancers direct expression to the Arcuate Nucleus and both must be deleted in mice to produce a dramatic change in expression and phenotype. $\mathbf{c}$ Enhancer turnover is when one enhancer disappears and a new one appears in the same regulatory region, replacing the lost function. In the example shown by Domené et al. [72], turnover resulted in no net change in expression or phenotype

can be evaluated in only one or a small number of embryonic stages, and not in adult tissues. Although we found that many HARs appear to function during development [10], a substantial fraction likely cannot be characterized with this approach. Generation of stable transgenic lines (where the F1 generation is analyzed) could facilitate the analysis of many more developmental stages and adult expression. However, the low throughput of transgenic experiments has limited their utility for characterizing large catalogs of human-specific regulatory elements.

Despite these caveats, a number of human-specific noncoding sequences have been functionally characterized as enhancers in vivo with reporter assays in transgenic animal models. These include two conserved elements deleted in humans (hCONDELs) [55], nearly 70 HARs (summarized in [10]), including nine haDHSs, and an additional seven haDHSs that do not overlap HARs [38]. Reporter assays in transgenic animals can also be used to test the hypothesis that human-specific mutations in non-coding elements altered their enhancer activity, and indeed several HARs and haDHSs show expression differences between constructs carrying the chimpanzee versus human sequence [37, 56-59] (Table 1). It is important to note that transient transgenics can capture gains and losses of enhancer activity in specific tissues or cell types (expression patterns), but they are not quantitative (expression level depends on number and location of random genomic integrations) and hence are unable to capture changes in activity levels. These studies aiming to analyze the function of humanspecific non-coding sequences and then to study them comparatively with transgenic model organism enhancer assays are adding valuable information about the functional impact of human-specific DNA changes. Looking ahead, it will be important to supplement these with additional approaches to link enhancer activity differences to gene expression and phenotypes.

Massively parallel reporter assays (MPRAs) show promise in cell lines and may become practical in whole animals [60-62]. In this technique, a library of candidate regulatory enhancer DNA sequences is cloned into a reporter construct containing a unique DNA barcode that will be transcribed if the enhancer is active. RNAsequencing of the DNA barcodes enables quantitative measurements of enhancer activity, including effects of individual nucleotide variants [62]. Two potential limitations of this technique are length of the candidate enhancers ( $<200$ bp with current DNA synthesis methods) and the fact that they are usually evaluated outside of their genome context. Knock-in strategies where regulatory element variants are replaced in the analyzed locus (such as via CRISPR/Cas9 genome editing) could address this issue. Low throughput transgenic and mutant model animals can complement both techniques. A combination of methods can together enable tests of hypotheses about the effects of human-specific variants on molecular and organismal phenotypes.

\section{Human variation links accelerated regions to phenotypes}

Another way to associate human-specific non-coding regions with traits is to investigate associations between any polymorphisms they harbor and phenotypic variability in humans. The polymorphic sites will be relatively recent mutations that are largely distinct from differences between human and chimpanzee reference genomes. But they may nonetheless shed light on the general function of the accelerated regions in which they occur. For example, gene copy number variants in the human-specific pericentric inversion of chromosome 1 have been associated with 
human developmental and neurogenetic diseases $[15,52$, 63-65], and we showed that HARs that are conserved in primates but not across all mammals are associated with schizophrenia [66]. As many more human genomes are sequenced, population genetic variation in and near to HARs will also help researchers to test hypotheses about the evolutionary forces that created and maintain HARs (Box 1, Fig. 3).

Leveraging this approach, Doan et al. sequenced HARs in individuals with autism spectrum disorder (ASD) and unaffected controls [60]. ASD was associated with a significant $43 \%$ excess of rare biallelic variants in HARs. The authors showed that $29 \%$ of these variants alter enhancer activity in primary mouse neurospheres using a custom MPRA. They further identified rare homozygous mutations with active regulatory marks near neurodevelopmental and disease-associated genes in ASD patients who lack causal coding mutations. Using luciferase reporter assays and transgenic mice, the authors show that one particular ASD-associated HAR variant (HACNS426) that was previously shown to interact with the neuronal morphology gene Cux1 [67-70] is an enhancer (Fig. 4). The authors report that transgenic mice carrying the mutant allele fused to the CUX1 promoter and to GFP show elevated expression of the reporter gene in the developing brain compared to the wild-type allele. But it is important to remember that transgenic enhancer assays cannot capture quantitative expression differences (see above).

The study of Doan et al. demonstrates the potential for human sequencing studies coupled with deep phenotyping to shed light on HAR function. Using associations between specific HARs and ASD, the authors conclude that many HARs are essential for normal development. Even though the ASD-associated mutations are different from the substitutions that happened during human evolution, they nonetheless help to annotate the function of the genomic region containing a HAR. Polymorphic deletions of HARs could similarly be used to annotate their functions. Doan et al. further hypothesize that human-specific mutations in HARs could have altered social and/or cognitive behavior. To test this hypothesis precisely with medical resequencing requires that specific human-chimp differences and combinations thereof be polymorphic in living humans or primates. As targeted sequencing (for example, with molecular inversion probes [71]) enables HARs to be sequenced in millions of individuals, it will become clear how many human-chimp differences are present in modern humans. One caveat is that the genomes of modern

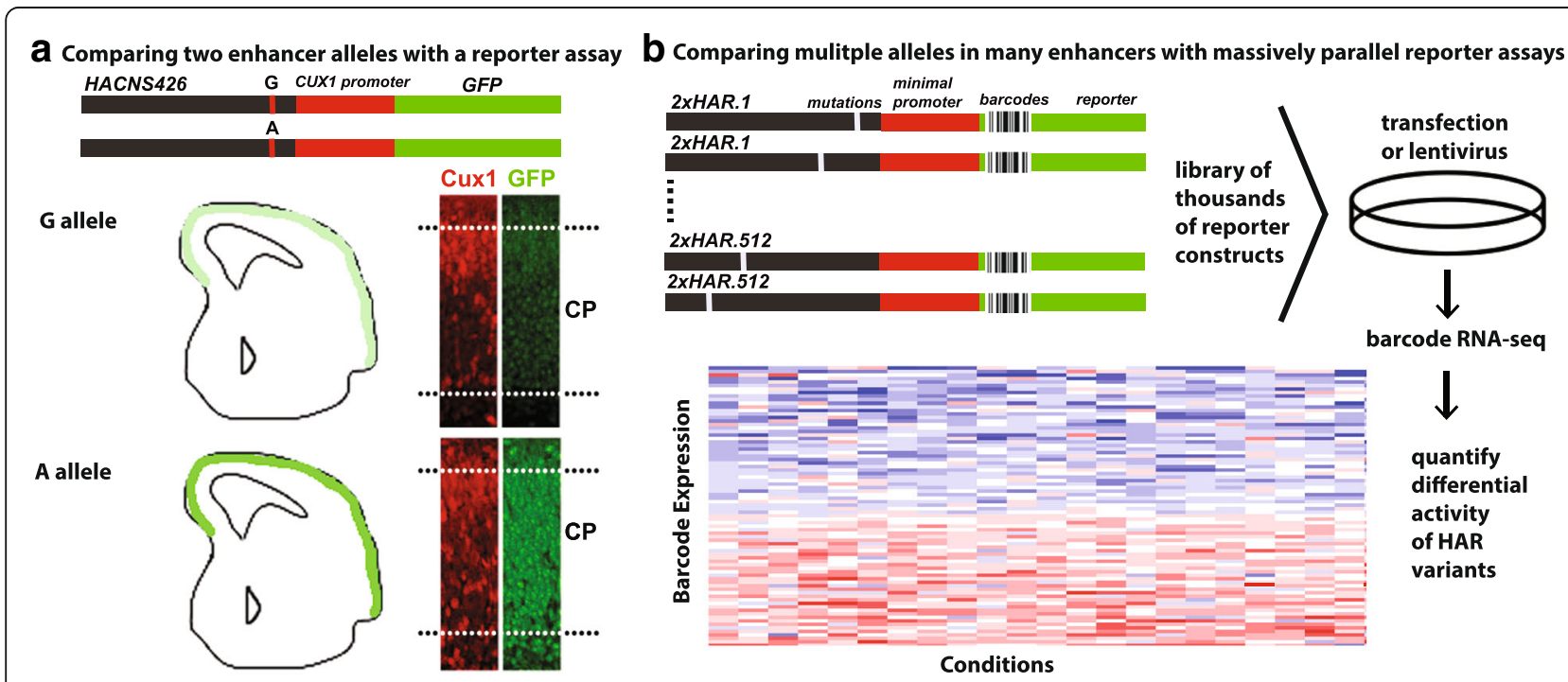

Fig. 4 Testing HARs and HAR sequence variants for enhancer activity with reporter assays. a Example of a transient transgenic reporter assay to test a HAR (HACNS426) for enhancer activity in mouse embryos. The experiment compares enhancer activity of the major allele (G) to that of the autism-associated minor allele (A) that is never homozygous in healthy controls and is predicted to change transcription factor binding. Top: constructs carrying each of the two HACNS426 alleles fused to the human CUX1 promoter and the GFP reporter gene are separately injected into single-cell mouse embryos. Bottom: to assay enhancer activity, brain slices from embryonic day E16.5 are stained for GFP. The authors observed differences in GFP expression with the G allele (above) versus A allele (below). The major strength of the approach is the spatial and cellular resolution of in vivo measurements, while weaknesses include not being highly quantitative, the use of mouse to compare human and chimpanzee variants, low throughput, and relatively high cost. Adapted with permission from [23]. The study also performed in vitro luciferase reporter assays and showed HANCS426 interacts with the dosage-sensitive CUX1 promoter. b Massively parallel reporter assays (MPRAs) enable thousands of reporter constructs to be tested as a library (top) in which each HAR variant is associated with a unique DNA barcode (such as 20-bp sequence). RNA sequencing of barcodes (bottom) provides a quantitative estimate of the activity of each HAR variant. MPRAs are high throughput, allowing thousands of HARs and variants thereof to be tested, and they are quantitative, enabling detection of single nucleotide differences with moderate effects on expression. Current weaknesses of the technology include being limited to HARs or HAR segments less than $200 \mathrm{bp}$ and being restricted to testing in cell lines or mouse tail vein assays 
humans and other primates differ from those of ancestral populations, so phenotype associations of HAR mutations must be interpreted in the context of the modern genetic background. For this approach to be a useful path to elucidating HAR functions in humans, it will also be important to couple sequencing data with a very wide range of reliably measured phenotypes ranging from diseases to behaviors. This is an exciting and increasingly feasible direction.

\section{Changing things so everything stays the same? Regulatory element turnover and compensatory evolution}

To measure the effects of non-coding mutations on expression and phenotypes, we must move towards considering the whole regulatory region of a gene, as well as changes in trans (such as, expression and activity of upstream regulators). One reason for this is that regulatory elements without sufficient sequence similarity to be aligned across species can direct conserved expression patterns, a phenomenon known as 'enhancer turnover' [72-74] (Fig. 3). Another consideration is the fact that regulatory elements interact with each other to drive gene expression, which means that non-coding mutations can amplify or buffer one another even if they are not adjacent. One particular type of compensation across regulatory elements involves 'shadow enhancers', which are distinct sequences capable of guiding similar spatiotemporal expression patterns [75-80] (Fig. 3). This apparently redundant function may ensure robust, precise, and stable regulation $[77,78]$. Thus, gene expression evolution cannot be easily predicted from a single human-specific regulatory element without evaluating the complete regulatory landscape of the locus.

Most human-specific regulatory elements have nonetheless been characterized one at a time, or even one mutation at a time. Supporting the idea that this limits our ability to predict expression divergence, Vermunt et al. showed that many loci contain both gains and losses of predicted enhancers (4.9-10.3\% across brain regions of mice, macaques, chimpanzees, and humans) [43]. For example, the locus containing HAR87 harbors multiple other predicted enhancers with increased or decreased activity during human evolution, measured via chromatin capture coverage (Fig. 2). These other changes could compensate for or otherwise interact with changes in the activity of HAR87. To investigate this possibility, comparative genomics should be combined with functional genomics assays, especially those that do not depend on antibody affinities (for example, chromatin capture, open chromatin).

An emerging approach is to leverage high-throughput genetic screens that can probe combinations of mutations across a locus, for example, via CRISPR genome editing [81, 82]. Using a combination of CRISPR activation and inhibition, as well as knock-outs and knock-ins, it will be possible to study the effect of human-chimpanzee noncoding sequence differences on gene expression in cells or in models such as mice. These studies should investigate all the putative regulatory regions of a gene individually and in combination to truly decipher the net regulatory effects.

\section{Thinking again: what are we missing in the study of genetic bases of human evolution?}

The past decade has seen significant progress towards addressing the major hurdles of associating human-specific non-coding elements with traits that make our species unique. Functional genomics has been transformative in terms of annotating and prioritizing HARs, and it has also been used directly to identify novel human-specific regulatory elements that are not divergent enough in sequence to be detectable in genome-wide tests for accelerated sequence evolution. As the mechanisms of gene regulation are increasingly understood, the functional effects of human-specific non-coding mutations are becoming less mysterious. High-throughput functional assays, such as massively parallel reporter assays and CRISPR screens, are increasing the numbers of characterized human-specific regulatory elements by orders of magnitude. Meanwhile, targeted and whole genome sequencing of thousands of people is enabling rare variant association and linkage studies that connect non-coding elements to traits and may even reveal the phenotypes of individuals carrying specific ancestral versus derived haplotypes. Human evolutionary studies will always be challenged by our inability to test specific hypotheses about genetic changes in the correct organismal context (an ancestral human). However, we now have a much broader collection of complementary research tools to address this problem, including on one hand genome editing of stem cell-derived cell lines, organoids, human primary cells, and model organisms, and on the other hand deep phenotyping and sequencing of humans and other primates to capture natural variation.

Despite these exciting advances towards understanding the role of regulatory changes in human evolution, it is important to ask what hurdles still remain. One major barrier is our very limited ability to assay functions of regulatory elements other than enhancers. Genome editing is helping to address this bias by enabling researchers to introduce individual human mutations or to knock out single humanspecific elements [81, 82]. By performing these experiments in primate cells, human cells, and model organisms, it will be feasible to characterize putative human-specific insulating and repressing elements in terms of their downstream effects on molecular and cellular phenotypes. The development of new high-throughput assays to read out regulatory functions other than enhancing gene expression (such as repression or insulation) will help as well. 
Another missing piece in the story of human regulatory evolution is the vast sequence differences encoded within SVs, many of which are missing or incorrect in human and non-human primate reference genomes [83]. Even within the SVs that have been accurately compared across primates, human non-coding evolution is relatively unexplored, due largely to the technical challenges of functionally testing regions with duplications and complex genomic architectures. SVs have immense potential to alter regulatory elements and their interactions with target genes. For example, inserting or deleting topologically associating domain (TAD) boundaries, which are often conserved across cell types and species [84], can associate regulatory elements with new genes (termed 'enhancer hijacking') or insulate them from their ancestral gene targets $[85,86]$. It will be exciting to explore the evolution of human gene expression in terms of 3D genome organization and its effects on regulatory interactions.

\section{Outlook: what will it take to crack the code?}

The next decade could be the one in which the regulatory code is cracked, opening the door to reading out the functional effects of non-coding changes that distinguish humans from other primates. To realize this goal, we should not only push to characterize the humanchimp differences for which we already have functional hypotheses. We must also continue to ask 'what genomic regions are missing from our analyses?' and 'where do current models of gene regulation fail to explain divergently expressed genes?'

\section{Authors' contributions}

LFF and KSP prepared figures and tables and wrote and revised the article. Both authors have read and agreed with the content.

\section{Acknowledgements}

This work was supported by grants from the Agencia Nacional de Promoción Científica y Tecnológica (PICT2013-1642; PICT 2015-1726) to LFF and by Gladstone Institutes to KSP.

\section{Competing interests}

The authors declare that they have no competing interests.

\section{Author details}

'Instituto de Investigaciones en Ingeniería Genética y Biología Molecular (INGEBI), Consejo Nacional de Investigaciones Científicas y Técnicas (CONICET), Buenos Aires, Argentina. ${ }^{2}$ Gladstone Institutes, San Francisco, CA 94158, USA. ${ }^{3}$ Department of Epidemiology \& Biostatistics, Institute for Human Genetics, Institute for Computational Health Sciences, University of California, San Francisco, CA 94158, USA.

Published online: 02 October 2017

\section{References}

1. Consortium HGS. Finishing the euchromatic sequence of the human genome. Nature. 2004;431:931-45.

2. Lander ES, Linton LM, Birren B, Nusbaum C, Zody MC, Baldwin J, et al. Initial sequencing and analysis of the human genome. Nature. 2001;409:860-921.

3. Consortium CSaA. Initial sequence of the chimpanzee genome and comparison with the human genome. Nature. 2005;437:69-87.
4. Gibbs RA, Rogers J, Katze MG, Bumgarner R, Weinstock GM, Mardis ER, et al. Evolutionary and biomedical insights from the rhesus macaque genome. Science. 2007;316:222-34.

5. King MC, Wilson AC. Evolution at two levels in humans and chimpanzees. Science. 1975;188:107-16.

6. Nuttall. Blood immunity and blood relationships. Cambridge, UK: Cambridge University Press; 1904.

7. Carroll SB. Evo-devo and an expanding evolutionary synthesis: a genetic theory of morphological evolution. Cell. 2008;134:25-36.

8. Enard W. Human evolution: enhancing the brain. Curr Biol. 2015;25:R421-3.

9. Franchini LF, Pollard KS. Can a few non-coding mutations make a human brain? Bioessays. 2015;37:1054-61.

10. Franchini LF, Pollard KS. Genomic approaches to studying human-specific developmental traits. Development. 2015;142:3100-12.

11. Reilly SK, Noonan JP. Evolution of gene regulation in humans. Annu Rev Genomics Hum Genet. 2016;17:45-67.

12. Silver DL. Genomic divergence and brain evolution: How regulatory DNA influences development of the cerebral cortex. Bioessays. 2016;38:162-71.

13. Enard W. The molecular basis of human brain evolution. Curr Biol. 2016;26: R1109-R17.

14. Hubisz MJ, Pollard KS. Exploring the genesis and functions of Human Accelerated Regions sheds light on their role in human evolution. Curr Opin Genet Dev. 2014:29:15-21.

15. O'Bleness M, Searles VB, Varki A, Gagneux P, Sikela JM. Evolution of genetic and genomic features unique to the human lineage. Nat Rev Genet. 2012; 13:853-66.

16. Preuss TM. Human brain evolution: from gene discovery to phenotype discovery. Proc Natl Acad Sci U S A. 2012;109 Suppl 1:10709-16.

17. Vallender EJ, Mekel-Bobrov N, Lahn BT. Genetic basis of human brain evolution. Trends Neurosci. 2008;31:637-44.

18. Pollard KS, Salama SR, Lambert N, Lambot MA, Coppens S, Pedersen JS, et al. An RNA gene expressed during cortical development evolved rapidly in humans. Nature. 2006;443:167-72.

19. Pollard KS, Salama SR, King B, Kern AD, Dreszer T, Katzman S, et al. Forces shaping the fastest evolving regions in the human genome. PLoS Genet. 2006;2, e168.

20. Bird CP, Stranger BE, Liu M, Thomas DJ, Ingle CE, Beazley C, et al. Fast-evolving noncoding sequences in the human genome. Genome Biol. 2007;8:R118.

21. Bush EC, Lahn BT. A genome-wide screen for noncoding elements important in primate evolution. BMC Evol Biol. 2008:8:17.

22. Lowe CB, Haussler D. 29 mammalian genomes reveal novel exaptations of mobile elements for likely regulatory functions in the human genome. PLoS One. 2012; , e43128.

23. Prabhakar S, Noonan JP, Paabo S, Rubin EM. Accelerated evolution of conserved noncoding sequences in humans. Science. 2006;314:786.

24. Pennacchio LA, Ahituv N, Moses AM, Prabhakar S, Nobrega MA, Shoukry M, et al. In vivo enhancer analysis of human conserved non-coding sequences. Nature. 2006;444:499-502.

25. Visel A, Blow MJ, Li Z, Zhang T, Akiyama JA, Holt A, et al. ChlP-seq accurately predicts tissue-specific activity of enhancers. Nature. 2009;457:854-8.

26. Visel A, Prabhakar S, Akiyama JA, Shoukry M, Lewis KD, Holt A, et al. Ultraconservation identifies a small subset of extremely constrained developmental enhancers. Nat Genet. 2008;40:158-60.

27. Dickel DE, Visel A, Pennacchio LA. Functional anatomy of distant-acting mammalian enhancers. Philos Trans R Soc Lond B Biol Sci. 2013;368: 20120359

28. Yunis JJ, Prakash O. The origin of man: a chromosomal pictorial legacy. Science. 1982;215:1525-30

29. Marques-Bonet T, Eichler EE. The evolution of human segmental duplications and the core duplicon hypothesis. Cold Spring Harb Symp Quant Biol. 2009;74:355-62.

30. Sudmant PH, Huddleston J, Catacchio CR, Malig M, Hillier LW, Baker C, et al. Evolution and diversity of copy number variation in the great ape lineage. Genome Res. 2013;23:1373-82.

31. Dennis MY, Eichler EE. Human adaptation and evolution by segmental duplication. Curr Opin Genet Dev. 2016:41:44-52.

32. Kostka D, Hahn MW, Pollard KS. Noncoding sequences near duplicated genes evolve rapidly. Genome Biol Evol. 2010;2:518-33.

33. Lupianez DG, Kraft K, Heinrich V, Krawitz P, Brancati F, Klopocki E, et al. Disruptions of topological chromatin domains cause pathogenic rewiring of gene-enhancer interactions. Cell. 2015;161:1012-25. 
34. Symmons O, Pan L, Remeseiro S, Aktas T, Klein F, Huber W, et al. The Shh topological domain facilitates the action of remote enhancers by reducing the effects of genomic distances. Dev Cell. 2016;39:529-43.

35. Consortium EP. An integrated encyclopedia of DNA elements in the human genome. Nature. 2012;489:57-74.

36. Ponting CP. Biological function in the twilight zone of sequence conservation. BMC Biol. 2017;15:71.

37. Capra JA, Erwin GD, McKinsey G, Rubenstein JL, Pollard KS. Many human accelerated regions are developmental enhancers. Philos Trans R Soc Lond B Biol Sci. 2013;368:20130025

38. Gittelman RM, Hun E, Ay F, Madeoy J, Pennacchio L, Noble WS, et al. Comprehensive identification and analysis of human accelerated regulatory DNA. Genome Res. 2015;25:1245-55.

39. Dong X, Wang X, Zhang F, Tian W. Genome-wide identification of regulatory sequences undergoing accelerated evolution in the human genome. Mol Biol Evol. 2016;33:2565-75.

40. Consortium EP, Birney E, Stamatoyannopoulos JA, Dutta A, Guigo R, Gingeras TR, et al. Identification and analysis of functional elements in $1 \%$ of the human genome by the ENCODE pilot project. Nature. 2007;447:799-816.

41. Cotney J, Leng J, Yin J, Reilly SK, DeMare LE, Emera D, et al. The evolution of lineage-specific regulatory activities in the human embryonic limb. Cell. 2013;154:185-96.

42. Reilly SK, Yin J, Ayoub AE, Emera D, Leng J, Cotney J, et al. Evolutionary genomics. Evolutionary changes in promoter and enhancer activity during human corticogenesis. Science. 2015;347:1155-9.

43. Vermunt MW, Tan SC, Castelijns B, Geeven G, Reinink P, de Bruijn E, et al. Epigenomic annotation of gene regulatory alterations during evolution of the primate brain. Nat Neurosci. 2016;19:494-503.

44. Prescott SL, Srinivasan R, Marchetto MC, Grishina I, Narvaiza I, Selleri L, et al. Enhancer divergence and cis-regulatory evolution in the human and chimp neural crest. Cell. 2015;163:68-83.

45. Bajpai R, Chen DA, Rada-Iglesias A, Zhang J, Xiong Y, Helms J, et al. CHD7 cooperates with PBAF to control multipotent neural crest formation. Nature. 2010:463:958-62

46. Rada-Iglesias A, Bajpai R, Swigut T, Brugmann SA, Flynn RA, Wysocka J. A unique chromatin signature uncovers early developmental enhancers in humans. Nature. 2011;470:279-83.

47. Doolittle WF. Is junk DNA bunk? A critique of ENCODE. Proc Natl Acad Sci U S A. 2013;110:5294-300.

48. Graur D, Zheng Y, Price N, Azevedo RB, Zufall RA, Elhaik E. On the immortality of television sets: 'function' in the human genome according to the evolution-free gospel of ENCODE. Genome Biol Evol. 2013;5:578-90.

49. Sakabe NJ, Nobrega MA. Beyond the ENCODE project: using genomics and epigenomics strategies to study enhancer evolution. Philos Trans R Soc Lond B Biol Sci. 2013;368:20130022.

50. Rossant J. Mouse and human blastocyst-derived stem cells: vive les differences. Development. 2015;142:9-12.

51. Florio M, Albert M, Taverna E, Namba T, Brandl H, Lewitus E, et al. Humanspecific gene ARHGAP11B promotes basal progenitor amplification and neocortex expansion. Science. 2015;347(6229):1465-70.

52. Charrier C, Joshi K, Coutinho-Budd J, Kim JE, Lambert N, de Marchena J, et al. Inhibition of SRGAP2 function by its human-specific paralogs induces neoteny during spine maturation. Cell. 2012;149:923-35.

53. Schreiweis C, Bornschein U, Burguiere E, Kerimoglu C, Schreiter S, Dannemann M, et al. Humanized Foxp2 accelerates learning by enhancing transitions from declarative to procedural performance. Proc Natl Acad Sci U S A. 2014;111:14253-8.

54. Enard W, Gehre S, Hammerschmidt K, Holter SM, Blass T, Somel M, et al. A humanized version of Foxp2 affects cortico-basal ganglia circuits in mice. Cell. 2009;137:961-71.

55. McLean CY, Reno PL, Pollen AA, Bassan Al, Capellini TD, Guenther C, et al. Human-specific loss of regulatory DNA and the evolution of human-specific traits. Nature. 2011:471:216-9.

56. Boyd JL, Skove SL, Rouanet JP, Pilaz $\sqcup$, Bepler T, Gordan R, et al. Humanchimpanzee differences in a FZD8 enhancer alter cell-cycle dynamics in the developing neocortex. Curr Biol. 2015;25:772-9.

57. Kamm GB, Lopez-Leal R, Lorenzo JR, Franchini LF. A fast-evolving human NPAS3 enhancer gained reporter expression in the developing forebrain of transgenic mice. Philos Trans R Soc Lond B Biol Sci. 2013;368:20130019.

58. Kamm GB, Pisciottano F, Kliger R, Franchini LF. The developmental brain gene NPAS3 contains the largest number of accelerated regulatory sequences in the human genome. Mol Biol Evol. 2013;30:1088-102.
59. Prabhakar S, Visel A, Akiyama JA, Shoukry M, Lewis KD, Holt A, et al. Humanspecific gain of function in a developmental enhancer. Science. 2008:321:1346-50.

60. Doan RN, Bae Bl, Cubelos B, Chang C, Hossain AA, Al-Saad S, et al. Mutations in human accelerated regions disrupt cognition and social behavior. Cell. 2016;167:341-54. e12.

61. Melnikov A, Murugan A, Zhang X, Tesileanu T, Wang L, Rogov P, et al. Systematic dissection and optimization of inducible enhancers in human cells using a massively parallel reporter assay. Nat Biotechnol. 2012;30:271-7.

62. Patwardhan RP, Hiatt JB, Witten DM, Kim MJ, Smith RP, May D, et al. Massively parallel functional dissection of mammalian enhancers in vivo. Nat Biotechnol. 2012;30:265-70.

63. Fortna A, Kim Y, MacLaren E, Marshall K, Hahn G, Meltesen L, et al. Lineagespecific gene duplication and loss in human and great ape evolution. PLoS Biol. 2004;2, E207.

64. Davis JM, Searles VB, Anderson N, Keeney J, Dumas L, Sikela JM. DUF1220 dosage is linearly associated with increasing severity of the three primary symptoms of autism. PLoS Genet. 2014;10, e1004241.

65. Dennis MY, Nuttle X, Sudmant PH, Antonacci F, Graves TA, Nefedov M, et al. Evolution of human-specific neural SRGAP2 genes by incomplete segmental duplication. Cell. 2012;149:912-22.

66. Xu K, Schadt EE, Pollard KS, Roussos P, Dudley JT. Genomic and network patterns of schizophrenia genetic variation in human evolutionary accelerated regions. Mol Biol Evol. 2015;32:1148-60.

67. Cubelos B, Sebastian-Serrano A, Beccari L, Calcagnotto ME, Cisneros E, Kim S, et al. Cux1 and Cux2 regulate dendritic branching, spine morphology, and synapses of the upper layer neurons of the cortex. Neuron. 2010;66:523-35.

68. Fullwood MJ, Han Y, Wei CL, Ruan X, Ruan Y. Chromatin interaction analysis using paired-end tag sequencing. Curr Protoc Mol Biol. 2010;Chapter 21 : Unit $21151-25$.

69. Grueber WB, Jan LY, Jan YN. Different levels of the homeodomain protein cut regulate distinct dendrite branching patterns of Drosophila multidendritic neurons. Cell. 2003;112:805-18.

70. Li G, Fullwood MJ, Xu H, Mulawadi FH, Velkov S, Vega V, et al. ChIA-PET tool for comprehensive chromatin interaction analysis with paired-end tag sequencing. Genome Biol. 2010;11:R22.

71. Cantsilieris S, Stessman HA, Shendure J, Eichler EE. Targeted Capture and high-throughput sequencing using molecular inversion probes (MIPs). Methods Mol Biol. 2017;1492:95-106.

72. Domene S, Bumaschny VF, de Souza FS, Franchini LF, Nasif S, Low MJ, et al. Enhancer turnover and conserved regulatory function in vertebrate evolution. Philos Trans R Soc Lond B Biol Sci. 2013;368:20130027.

73. Harmston N, Baresic A, Lenhard B. The mystery of extreme non-coding conservation. Philos Trans R Soc Lond B Biol Sci. 2013;368:20130021.

74. Maeso I, Irimia M, Tena JJ, Casares F, Gomez-Skarmeta JL. Deep conservation of cis-regulatory elements in metazoans. Philos Trans R Soc Lond B Biol Sci. 2013;368:20130020.

75. de Souza FS, Franchini LF, Rubinstein M. Exaptation of transposable elements into novel cis-regulatory elements: is the evidence always strong? Mol Biol Evol. 2013:30:1239-51.

76. Franchini LF, Lopez-Leal R, Nasif S, Beati P, Gelman DM, Low MJ, et al. Convergent evolution of two mammalian neuronal enhancers by sequential exaptation of unrelated retroposons. Proc Natl Acad Sci U S A. 2011;108(37):15270-5.

77. Frankel N, Davis GK, Vargas D, Wang S, Payre F, Stern DL. Phenotypic robustness conferred by apparently redundant transcriptional enhancers. Nature. 2010;466:490-3.

78. Lam DD, de Souza FS, Nasif S, Yamashita M, Lopez-Leal R, Otero-Corchon V, et al. Partially redundant enhancers cooperatively maintain Mammalian pomc expression above a critical functional threshold. PLoS Genet. 2015;11, e1004935.

79. Levine M. Transcriptional enhancers in animal development and evolution. Curr Biol. 2010;20:R754-63.

80. Rubinstein $M$, de Souza FS. Evolution of transcriptional enhancers and animal diversity. Philos Trans R Soc Lond B Biol Sci. 2013;368:20130017.

81. Gilbert LA, Horlbeck MA, Adamson B, Villalta JE, Chen Y, Whitehead EH, et al. Genome-scale CRISPR-mediated control of gene repression and activation. Cell. 2014;159:647-61.

82. Zheng Q, Cai X, Tan MH, Schaffert S, Arnold CP, Gong X, et al. Precise gene deletion and replacement using the CRISPR/Cas9 system in human cells. Biotechniques. 2014:57:115-24.

83. Cheng Z, Ventura M, She X, Khaitovich P, Graves T, Osoegawa K, et al. A genome-wide comparison of recent chimpanzee and human segmental duplications. Nature. 2005;437:88-93. 
84. Vietri Rudan M, Barrington C, Henderson S, Ernst C, Odom DT, Tanay A, et al. Comparative Hi-C reveals that CTCF underlies evolution of chromosomal domain architecture. Cell Rep. 2015;10:1297-309.

85. Acemel RD, Maeso I, Gomez-Skarmeta JL. Topologically associated domains: a successful scaffold for the evolution of gene regulation in animals. Wiley Interdiscip Rev Dev Biol. 2017;6.

86. Maeso I, Acemel RD, Gomez-Skarmeta JL. Cis-regulatory landscapes in development and evolution. Curr Opin Genet Dev. 2017:43:17-22.

87. Pollard KS, Hubisz MJ, Rosenbloom KR, Siepel A. Detection of nonneutral substitution rates on mammalian phylogenies. Genome Res. 2010;20:110-21.

88. Kostka D, Hubisz MJ, Siepel A, Pollard KS. The role of GC-biased gene conversion in shaping the fastest evolving regions of the human genome. Mol Biol Evol. 2012;29:1047-57.

89. Katzman S, Kern AD, Pollard KS, Salama SR, Haussler D. GC-biased evolution near human accelerated regions. PLoS Genet. 2010;6, e1000960.

90. Sumiyama K, Saitou N. Loss-of-function mutation in a repressor module of human-specifically activated enhancer HACNS1. Mol Biol Evol. 2011;28:3005-7.

91. Sikela JM. The jewels of our genome: the search for the genomic changes underlying the evolutionarily unique capacities of the human brain. PLoS Genet. 2006;2, e80

92. Varki A, Altheide TK. Comparing the human and chimpanzee genomes: searching for needles in a haystack. Genome Res. 2005;15:1746-58.

93. Herculano-Houzel S. The remarkable, yet not extraordinary, human brain as a scaled-up primate brain and its associated cost. Proc Natl Acad Sci U S A. 2012;109 Suppl 1:10661-8.

94. Preuss TM. Primate brain evolution in phylogenetic context. In: Kaas J, editor. Evolution of nervous systems. 4. Oxford, UK: Elsevier; 2007. p. 1-34.

95. Semendeferi K, Lu A, Schenker N, Damasio H. Humans and great apes share a large frontal cortex. Nat Neurosci. 2002;5:272-6.

96. Gabi M, Neves K, Masseron C, Ribeiro PF, Ventura-Antunes L, Torres $L$, et al. No relative expansion of the number of prefrontal neurons in primate and human evolution. Proc Natl Acad Sci U S A. 2016;113:9617-22.

97. Semendeferi K, Teffer K, Buxhoeveden DP, Park MS, Bludau S, Amunts K, et al. Spatial organization of neurons in the frontal pole sets humans apart from great apes. Cereb Cortex. 2011;21:1485-97.

98. Bianchi S, Stimpson CD, Duka T, Larsen MD, Janssen WG, Collins Z, et al. Synaptogenesis and development of pyramidal neuron dendritic morphology in the chimpanzee neocortex resembles humans. Proc Nat Acad Sci U S A. 2013;110 Suppl 2:10395-401.

99. Spocter MA, Hopkins WD, Barks SK, Bianchi S, Hehmeyer AE, Anderson SM, et al. Neuropil distribution in the cerebral cortex differs between humans and chimpanzees. J Comp Neurol. 2012;520:2917-29.

100. Lindblad-Toh K, Garber M, Zuk O, Lin MF, Parker BJ, Washietl S, et al. A highresolution map of human evolutionary constraint using 29 mammals. Nature. 2011;478:476-82.

101. Cook EC, Nelson JK, Sorrentino V, Koenis D, Moeton M, Scheij S, et al. Identification of the ER-resident E3 ubiquitin ligase RNF145 as a novel LXRregulated gene. PLoS One. 2017;12, e0172721. 